\title{
АНАЛИЗ ТЕХНИКО-ЭКОНОМИЧЕСКИХ ПОКАЗАТЕЛЕЙ И УДЕЛЬНОГО КОЭФФИЦИЕНТА ПОГЛОЩЕНИЯ ЭЛЕКТРОМАГНИТНОЙ ЭНЕРГИИ (SAR) СМАРТФОНОВ SAMSUNG
}

\author{
Кузнецова М.С.
}

В работе приведены результаты исследования зависимости удельного коэффициента поглощения электромагнитной энергии SAR от технико-экономических показателей смартфонов и иены от технических показателей и выявлены факторы, способствующих снижению SAR и росту иенын.

DOI: $10.20537 /$ mce2021econ13

Введение. Технические показатели (ТП) смартфонов Samsung постоянно улучшаются, поэтому представляет интерес анализ их динамики. При этом, необходимо учитывать электромагнитное излучение смартфонов, оказывающее негативное воздействие на здоровье человека. Смартфон - одно из самых распространенных устройств, излучающих энергию электромагнитного поля; является наглядным примером проблемной инновации, несущей не только пользу, но и вред [1, 2]. Даже международное агентство по изучению рака отнесло смартфоны к классу 2Б по канцероопасности (потенциальная опасность) [3, 4].

В связи с этим интерес также представляет анализ зависимости показателя, определяющего уровень энергии электромагнитного поля, которая поглощается в тканях тела человека (SAR, Вт/кг) от техникоэкономических показателей (ТЭП) смартфонов Samsung.

Анализ ТП смартфонов Samsung. Был проведен анализ тенденций изменения следующих технических показателей смартфонов: число ядер процессора, частота процессора, объем оперативной памяти, объем встроенной памяти, емкость аккумулятора, размер диагонали экрана и вес. По результатам исследования динамики технических показателей смартфонов выпуска 2011-2019 гг. были выделены этапы развития: 2011-2013 гг., 2014-2015 гг., 2016-2017 гг., 2018-2019 гг. 
Результаты анализа технических показателей смартфонов показали, что объем оперативной памяти положительно коррелирован с частотой процессора, объемом встроенной памяти; емкость аккумулятора положительно коррелирована с размером диагонали экрана и весом. Так, например, при увеличении объема оперативной памяти на 1 ГБ возрастала частота процессора на 0.2-0.5 ГГц, а при росте диагонали экрана на 1 дюйм увеличивалась емкость аккумулятора на 673-1430 мАч.

Анализ зависимости цены от ТП смартфонов. В табл. 1. приведены данные о приросте цены смартфонов Samsung 2011-2019 гг. выпуска при увеличении ТП на 1 ед.

Таблица 1. Прирост цены смартфонов Samsung 2011-2019 гг. выпуска при увеличении ТП на 1 ед., руб. за 1 ед., расчет по данным [5-12].

\begin{tabular}{|l|c|c|c|c|}
\hline \multicolumn{1}{|c|}{ Показатель } & \multicolumn{5}{|c|}{ Год выпуска смартфонов (гг.) } \\
\hline $\begin{array}{l}\text { Число ядер про- } \\
\text { цессора (ед) }\end{array}$ & - & $630-640$ & - & - \\
\hline $\begin{array}{l}\text { Частота процес- } \\
\text { сора (ГГц) }\end{array}$ & 1500 & - & $17720-22690$ & $32670-42200$ \\
\hline $\begin{array}{l}\text { Объем оператив- } \\
\text { ной памяти (ГБ) }\end{array}$ & 840 & $1190-1860$ & 4200 & 9870 \\
\hline $\begin{array}{l}\text { Объем встроен- } \\
\text { ной памяти (ГБ) }\end{array}$ & 70 & $90-140$ & $100-180$ & $40-80$ \\
\hline $\begin{array}{l}\text { Емкость аккуму- } \\
\text { лятора (мАч) }\end{array}$ & 1 & - & 10 & 10 \\
\hline $\begin{array}{l}\text { Размер диагонали } \\
\text { экрана (дм) }\end{array}$ & - & - & $16020-25420$ & 42070 \\
\hline Вес (г) & - & - & $380-660$ & - \\
\hline
\end{tabular}

Результаты анализа показали следующее: увеличение частоты процессора, объема оперативной памяти, емкости аккумулятора и размера диагонали экрана на каждом последующем этапе развития требовало более крупных денежных затрат; при этом, подобная ситуация наблюдалась с зависимостью цены от объема встроенной памяти у смартфонов выпуска 2011-2017 гг., а уже у моделей выпуска 20182019 гг. стоимость увеличения объема встроенной памяти составляла менее половины от значений прошлого периода, однако при этом при- 
рост цены смартфонов Samsung был значительным при увеличении частоты процессора (до 42200 руб. за 1 ГГц) и размера диагонали экрана (до 42070 руб. за 1 дм.).

Анализ зависимости SAR от TЭП смартфонов. Для разрешения продажи смартфонов значение удельного коэффициента поглощения электромагнитной энергии не должно превышать 2 Вт/кг. Уже при таком значении SAR может происходить некоторый нагрев кожи в месте контакта со смартфоном, а негативное воздействие свыше 4.5-6 Вт/кг способно привести к росту температуры тела [13]. Следует отметить, что один смартфон, допущенный к продаже, не может показать значение свыше 2 Вт/кг, но сейчас нередко используют сразу нескольких устройств, что увеличивает отрицательное воздействие на здоровье.

C ростом цены значение SAR возрастало для смартфонов выпуска 2014-2015 гг., 2018-2019 гг. и снижалось для смартфонов выпуска 2011-2013 гг., 2016-2017 гг.

При исследовании зависимости SAR от технико-экономических показателей были проверены 2 утверждения:

1) у более крупных смартфонов (коррелирующие показатели: размер диагонали экрана и вес) значение SAR меньше;

2) значение SAR снижается при повышении технических показателей у недорогих смартфонов и растет - при улучшении у более дорогих моделей.

Рассмотрение зависимости SAR от технических показателей проводилось как для четырех этапов развития (табл. 2), так и для групп смартфонов, выделенных из второго и третьего этапов. Из-за большого разброса цен у смартфонов выпуска 2014-2015 гг. и 2016-2017 гг. был проведен уточненный анализ для двух групп устройств: для выпуска 2014-2015 гг. - смартфоны, ценой до 10 тыс. руб. и более 10 тыс. руб. (табл. 3); для выпуска 2016-2017 гг. - смартфоны, ценой до 20 тыс. руб. и более 20 тыс. руб. (табл. 4).

Увеличение частоты процессора у смартфонов выпуска 20142015 гг., 2018-2019 гг. вызывало рост SAR. Также у смартфонов 20112013 гг., 2016-2019 гг. выпуска была определена отрицательная зависимость SAR от размеров смартфона (размер диагонали экрана, вес). Наибольшее снижение значения SAR вызывало увеличение размера диагонали экрана, что подтверждает первое утверждение. 
Анализ и моделирование экономических и сочиильньх процессов - МКО - 2021

Analysis and modeling of economic and social processes - MCE - 2021

Таблица 2. Прирост SAR смартфонов Samsung 2011-2019 гг. выпуска при увеличении ТП на 1 ед., Вт/кг за 1 ед., расчет по данным [5-12].

\begin{tabular}{|l|c|c|c|c|}
\hline & \multicolumn{4}{|c|}{ Год выпуска смартфонов (гг.) } \\
\hline \multicolumn{1}{|c|}{ Показатель } & $\begin{array}{c}\mathbf{2 0 1 1 -} \\
\mathbf{2 0 1 3}\end{array}$ & $\begin{array}{c}\mathbf{2 0 1 4}- \\
\mathbf{2 0 1 5}\end{array}$ & $\begin{array}{c}\mathbf{2 0 1 6} \\
\mathbf{2 0 1 7}\end{array}$ & $\mathbf{2 0 1 8 - 2 0 1 9}$ \\
\hline Число ядер процессора (ед.) & - & 0.02 & -0.06 & - \\
\hline Частота процессора (ГГц) & - & 0.15 & - & $0.07-0.16$ \\
\hline Объем оперативной памяти (ГБ) & - & - & -0.06 & 0.01 \\
\hline Объем встроенной памяти (ГБ) & -0.005 & 0.003 & - & - \\
\hline Емкость аккумулятора (мАч) & - & 0.00008 & -0.0003 & -0.00008 \\
\hline Размер диагонали экрана (дм) & -0.15 & - & -0.21 & $-0.11-0.23$ \\
\hline Вес смартфона (г) & - & - & - & $-0.002-0.003$ \\
\hline
\end{tabular}

Таблица 3. Прирост SAR для двух групп смартфонов Samsung (ценой до 10 тыс. руб. и более 10 тыс. руб.) 2014-2015 гг. выпуска при увеличении ТП на 1 ед., Вт/кг за 1 ед., расчет по данным [5-12].

\begin{tabular}{|l|c|c|}
\hline \multirow{2}{*}{\multicolumn{1}{|c|}{ Показатель }} & \multicolumn{2}{|c|}{ Смартфоны 2014-2015 гг. выпуска } \\
\cline { 2 - 3 } & $\begin{array}{c}\text { Ценой до 10 } \\
\text { тыс.руб. }\end{array}$ & $\begin{array}{c}\text { Ценой более } 10 \\
\text { тыс.руб. }\end{array}$ \\
\hline Число ядер процессора (ед.) & -0.08 & 0.03 \\
\hline Объем оперативной памяти (ГБ) & -0.25 & - \\
\hline Объем встроенной памяти (ГБ) & -0.01 & 0.001 \\
\hline Емкость аккумулятора (мАч) & -0.0004 & - \\
\hline
\end{tabular}

Таблица 4. Прирост SAR для двух групп смартфонов Samsung (ценой до 20 тыс. руб. и более 20 тыс. руб.) 2016-2017 гг. выпуска при увеличении ТП на 1 ед., Вт/кг за 1 ед., расчет по данным [5-12].

\begin{tabular}{|l|c|c|}
\hline \multirow{2}{*}{\multicolumn{1}{|c|}{ Показатель }} & \multicolumn{2}{|c|}{ Смартфоны 2016-2017 гг. выпуска } \\
\cline { 2 - 3 } & $\begin{array}{c}\text { Ценой до 20 } \\
\text { тыс.руб. }\end{array}$ & $\begin{array}{c}\text { Ценой более } 20 \\
\text { тыс.руб. }\end{array}$ \\
\hline Число ядер процессора (ед.) & -0.04 & - \\
\hline Частота процессора (ГГц) & -0.33 & $0.1-0.14$ \\
\hline Объем оперативной памяти (ГБ) & -0.08 & $-0.03-0.06$ \\
\hline Объем встроенной памяти (ГБ) & -0.01 & - \\
\hline Емкость аккумулятора (мАч) & $-0.0002-0.0003$ & $-0.0001-0.0002$ \\
\hline Размер диагонали экрана (дм) & $-0.3-0.49$ & $-0.17-0.27$ \\
\hline Вес смартфона (г) & - & $-0.002-0.004$ \\
\hline
\end{tabular}


Таким образом, результаты анализа зависимости SAR от техникоэкономических показателей смартфонов Samsung показали рост SAR c увеличением цены для смартфонов выпуска 2014-2015 гг. и 20182019 гг. Рост SAR также происходил при увеличении частоты процессора у смартфонов выпуска 2014-2015 гг., 2018-2019 гг. и выпуска 20162017 гг. при цене более 20 тыс. руб., объема оперативной памяти у смартфонов выпуска 2018-2019 гг., объема встроенной памяти у смартфонов выпуска 2014-2015 гг. моделей с ценой более 10 тыс. руб. Кроме того, SAR возрастал с ростом числа ядер процессора у смартфонов выпуска 2014-2015 гг. дороже 10 тыс. руб. и емкости аккумулятора у смартфонов этого поколения.

Снижение SAR происходило с ростом числа ядер у смартфонов выпуска 2014-2015 гг. для моделей с ценой до 10 тыс. руб. и выпуска 2016-2017 гг. для моделей с ценой до 20 тыс. руб., частоты процессора у смартфонов выпуска 2016-2017 гг. при цене до 20 тыс. руб., объема оперативной памяти у смартфонов выпуска 2016-2017 гг. и выпуска 2014-2015 гг. при цене до 10 тыс. руб. Также SAR снижался с увеличением объема встроенной памяти у смартфонов выпуска 2011-2013 гг., выпуска 2014-2015 гг. моделей с ценой до 10 тыс. руб. и выпуска 20162017 гг. при цене до 20 тыс. руб., емкости аккумулятора у смартфонов выпуска 2016-2017 гг., 2018-2019 гг. и выпуска 2014-2015 гг. моделей с ценой до 10 тыс. руб. Увеличение размера диагонали экрана вызывало снижение SAR у смартфонов выпуска 2011-2013 гг., 2016-2017 гг. и 2018-2019 гг., увеличение веса - у смартфонов выпуска 2018-2019 гг. и выпуска 2016-2017 гг. при цене более 20 тыс. руб.

Выводы. Исследование зависимости цены от технических показателей смартфонов Samsung выпуска 2011-2019 гг. показало, что цена смартфона существенно возрастает с увеличением частоты процессора и размера диагонали экрана.

У более дорогих смартфонов выпуска 2014-2015 гг. рост числа ядер процессора и объема встроенной памяти вызывал увеличение значения SAR, а у дешевых смартфонов - снижение. Также у дорогих смартфонов выпуска 2016-2017 гг. рост частоты процессора вызывал увеличение значения SAR, а у недорогих смартфонов - снижение. Таким образом, улучшение технических показателей недорогих смартфонов Samsung выпуска 2014-2015 гг. и 2016-2017 гг. уменьшало значение SAR. 
При этом с ростом размера диагонали экрана и веса смартфонов значение SAR снижается. Поэтому к более безопасным (в плане воздействия энергии электромагнитного поля) смартфонам Samsung выпуска 2011-2019 гг. можно отнести недорогие модели с большим размером диагонали экрана и весом.

\section{СПИСОК ЛИТЕРАТУРЫ}

1. Варшавский А.Е. Проблемные инновации: риски для человечества. М.: ЛЕНАНД, 2014.

2. Specific absorption rate / Wikipedia. URL: https://en.wikipedia.org/wiki/Specific_absorption_rate

3. Романова Ю. Просто SAR: насколько опасно радиочастотное излучение смартфонов для человека. Известия. Март 18, 2020.

URL: https:/iz.ru/986258/iuliia-romanova/prosto-sar-naskolko-opasnoradiochastotnoe-izluchenie-smartfonov-dlia-cheloveka

4. International Agency for Research on Cancer. IARC Report to the Union for International Cancer Control (UICC) on the Interphone Study. October 03, 2011. URL: https://interphone.iarc.fr/uicc-report-final-03102011.pdf

5. Bundesamt für Strahlenschutz. SAR Suche. URL: https:/www.bfs.de/SiteGlobals/Forms/Suche/BfS/DE/SARsuche_Formular.html ?gts $=6048912$ list\%253Dcsstring_SARManufacturer_sort\%252Basc

6. Смартфоны Samsung. Мвидео. URL: https://www.mvideo.ru/smartfony-isvyaz-10/smartfony-205/f/brand=samsung

7. Смартфоны Samsung. E-Katalog. URL: https://www.ekatalog.ru/list/122/samsung/

8. Смартфоны Samsung. Эльдорадо. URL: https://www.eldorado.ru/c/smartfony/b/SAMSUNG/

9. Смартфоны Samsung. Ситилинк. URL: https://www.citilink.ru/catalog/smartfony/?f=discount.any\%2C1376_214samsung

10. Смартфоны Samsung. Интернет-магазин MTC. URL: https://shop.mts.ru/catalog/smartfony/samsung/

11. Смартфоны Samsung. Билайн. URL: https:/moskva.beeline.ru/shop/catalog/telefony/smartfony/proizvoditel-samsung/

12. Смартфоны Samsung. DNS. URL: https://www.dnsshop.ru/catalog/17a8a01d16404e77/smartfony/?brand=samsung

13. Guidelines for limiting exposure to electromagnetic fields ( $100 \mathrm{kHz}$ to $300 \mathrm{GHz}) /$ ICNIRP. URL: https://www.icnirp.org/cms/upload/publications/ICNIRPrfgdl2020.pdf 


\section{ANALYSIS OF THE TECHNICAL AND ECONOMIC INDICATORS AND SPECIFIC ABSORPTION RATE OF ELECTROMAGNETIC ENERGY (SAR) OF SAMSUNG SMARTPHONES}

\section{Kuznetzova M.S.}

The paper aims at studying the dependence between the specific absorption rate of electromagnetic energy (SAR) and the technical-economic indicators of smartphones as far as between prices and the technical indicators, in order to determine factors that contribute to a decrease in SAR and an increase in prices. 